\title{
Cartographie des écosystèmes du Cameroun
}

\author{
Jean Michel ONANA \\ Université de Yaoundé I, Faculté des Sciences, Département de Biologie et Physiologie Végétales, \\ BP. 812 Yaoundé; Herbier National du Cameroun, B.P. 1601 Yaoundé, Cameroun. \\ E-mail : jeanmichelonan@gmail.com
}

\section{RÉSUMÉ}

Dans le cadre des recherches sur la diversité biologique du Cameroun, il a été constaté que sur les cartes présentées comme représentant les écosystèmes du Cameroun, tous les six écosystèmes ne sont pas représentés, aussi la dénomination des unités écosystémiques n'est pas celle des écosystèmes. C'est pourquoi l'objectif de ce travail était d'élaborer une carte des écosystèmes du Cameroun conforme à la nomenclature de la Convention sur la Diversité Biologique. Le matériel est constitué de cartes thématiques des caractères biophysiques du milieu. La méthode est d'utiliser des documents déjà publiés. L'analyse de la nomenclature des écosystèmes a permis d'identifier les six écosystèmes du Cameroun. Puis les couches des cartes thématiques ont été superposées pour cartographier les écosystèmes. C'est la carte phytogéographique qui est le modèle et la base de la délimitation des écosystèmes. Le résultat est la carte des six principaux écosystèmes: l'écosystème d'eau douce qui comprend les lacs naturels et artificiels, les milieux humides, les cours d'eau répartis dans tout le territoire; l'écosystème semi-aride réparti sur la plaine du Diamaré et une partie de la cuvette de la Bénoué ; l'écosystème de savane tropicale boisée sur la cuvette de la Bénoué, la plaine du nord, le plateau de l'Adamaoua, les hautes terres de l'Ouest et le nord du plateau méridional; l'écosystème de montagne qui est un archipel réparti sur les montagnes au-dessus de $1200 \mathrm{~m}$ au nord et $1500 \mathrm{~m}$ au sud réparti sur la ligne volcanique du Cameroun; l'écosystème de forêt tropicale dense humide qui occupe la plaine littorale et le plateau méridional ; et enfin l'écosystème marin et côtier localisé sur la côte et le plateau continental. La superficie approximative occupée par chaque écosystème a été calculée. En conclusion cette carte répond à une lacune par la documentation de la diversité biologique du Cameroun dans le volet de la connaissance des écosystèmes. C'est un outil pour la planification, la gestion durable des ressources biologiques, l'exercice de la souveraineté sur la biodiversité, l'enseignement et la recherche sur les ressources biologiques en tenant compte de tous les milieux naturels.

(C) 2018 International Formulae Group. All rights reserved.

Mots clés: Cameroun, caractères, carte, écosystème, nomenclature, biodiversité.

\section{Mapping the ecosystems of Cameroon}

\begin{abstract}
In the framework of the research on the biodiversity of Cameroon, it was found that on maps presenting the ecosystems of Cameroon, not all the six main ecosystems are represented, and the denomination of the ecosystem units is not that of ecosystems. The purpose of the present study was to develop a map of the ecosystems of Cameroon to accommodate these ecosystems to the nomenclature of the Convention on Biological Diversity. The material used consists of already published thematic maps of the biophysical characters of the environment. The analysis of the nomenclature of ecosystem typology made it possible to
\end{abstract}


identify the six ecosystems in Cameroon. Then the layers of the thematic maps were superimposed to map ecosystems. The phytogeographic map is the model and the basis of the delimitation of the ecosystems. The result is a map of the six main ecosystems in Cameroon: the freshwater ecosystems distributed throughout the territory, including natural and artificial lakes, wetlands, and water courses; the semi-arid ecosystem spread over the Diamaré plain and part of the Benoué basin; the wooded tropical savanna ecosystem on the Benoué basin, the northern plain, the Adamawa plateau, and the western and the northern sections of the Cameroon highlands; the montane ecosystem which is like an archipelago spread over the mountains above $1200 \mathrm{~m}$ in the north and $1500 \mathrm{~m}$ in the south, distributed across the volcanic line of Cameroon; the dense humid tropical forest ecosystem that occupies the coastal plain and the southern plateau; and finally the marine and coastal ecosystem located along the coast and the continental shelf. The approximate area of occupation of each ecosystem has been calculated. In conclusion, this map responds to a gap in the documentation of biological diversity in the ecosystem classification, by taking into account all the natural environments. It is a tool for planning, sustainable management of biological resources, exercise of sovereignty over biodiversity, teaching, and research on biological resources.

(C) 2018 International Formulae Group. All rights reserved.

Keywords: Cameroon, characters, map, ecosystem, nomenclature, biodiversity.

\section{INTRODUCTION}

Le Cameroun est situé en Afrique ouest-centrale entre les parallèles de latitude $01^{\circ} 40^{\prime}$ et $13^{\circ} 04^{\prime} \mathrm{N}$ et les méridiens de longitude $08^{\circ} 40^{\prime}$ et $16^{\circ} 10^{\prime} \mathrm{E}$; de forme générale triangulaire, il s'étend sur environ $800 \mathrm{~km}$ à la base et $1500 \mathrm{~km}$ sur la plus grande longueur et l'altitude atteint $4070 \mathrm{~m}$ de hauteur, pour une superficie d'environ $475.442 \mathrm{~km}^{2}$. Le territoire est connu pour la variété et la richesse de sa diversité floristique (Onana, 2010, 2015). Afin d'atteindre les objectifs du développement durable, le Gouvernement de la République a ratifié la Convention sur la Diversité Biologique (CDB) en 1994 et met en œuvre les engagements de la convention (Republic of Cameroon, 2009, 2012 ; République du Cameroun, 2014). Pour cela, la stratégie utilisée par la CDB pour tenter de freiner la perte de biodiversité est de promouvoir une "approche par écosystème". Il s'agit d'une stratégie qui soutient une gestion intégrée des ressources du sol, de l'eau et des ressources vivantes, afin d'encourager la conservation et l'utilisation durable réalisées d'une manière équitable (Secrétariat de la Convention sur la Diversité Biologique, 2004 ; CDB, 2012a, 2012b).

L'écosystème selon la CDB (1992) est " le complexe dynamique formé de communautés de plantes, d'animaux et de micro-organismes et de leur environnement non vivant qui par leur interaction, forment une unité fonctionnelle". Selon l'approche par écosystème, le terme "écosystème" renvoie à toute unité fonctionnelle, à quelque échelle que ce soit. La notion d'écosystème repose alors sur la relation qui existe entre un biotope (caractéristiques physico-chimiques d'un milieu) et les communautés vivantes qui le fréquentent (biocénoses).

On retrouve au Cameroun six écosystèmes (Republic of Cameroon, 2009 ; République du Cameroun, 2012, 2014): zone semi-aride, savane tropicale boisée, montagne, forêt tropicale dense humide, marin et côtier, et d'eau douce. Des cartes des écosystèmes ont été présentées dans les rapports quinquennaux de mise en œuvre de la convention (Republic of Cameroon, 2009; République du Cameroun, 2012). Malheureusement, sur les deux cartes, tous les six écosystèmes ne sont pas représentés. C'est le cas des écosystèmes d'eau douce, de montagne, et marins et côtiers. Enfin, dans République du Cameroun (2012), c'est la carte des zones agro-écologiques et administrative des structures de l'Institut de Recherche Agricole pour le Développement (IRAD) qui tient compte des activités agricoles suivant les caractéristiques du climat et secondairement du relief sur la ligne volcanique du Cameroun; aussi l'écosystème de forêt est divisé par la considération du rythme des saisons, selon que l'on a deux saisons (pluviométrie monomodale du climat camerounien) ou quatre saisons (pluviométrie bimodale du climat équatorial), qui 
correspondent plutôt aux phytochories des forêts tropicales denses humides toujours vertes atlantiques d'une part et de forêt dense humide semi-décidue d'autre part. A l'évidence donc, ces cartes ne peuvent être considérées comme représentant la distribution spatiale des écosystèmes du Cameroun.

C'est pourquoi, dans le cadre des recherches sur la diversité biologique du Cameroun, il a semblé nécessaire d'élaborer une carte des écosystèmes conforme à la nomenclature de la CDB. La base de ce travail est la carte des groupements végétaux du Cameroun, la végétation et la flore étant les meilleurs indicateurs de l'influence des facteurs biophysiques du milieu, y compris la faune qui est distribuée selon les types de végétation aussi bien dans le passé qu'actuellement (Amiet 1987, 2012 ; Vivien 2012 ; Vivien et Depierre, 2012) et des activités anthropiques (Lézine et al., 2013; Neumann et al., 2012) . C'est, en effet, à travers des études paléobotaniques qu'ont été établies les tendances des paléoenvironnements ou écosystèmes du passé (Achoundong, 1994 ; Lézine, 2008 ; Maley, 1987, 2001). Ce travail est justifié du fait que l'écosystème est l'unité fonctionnelle pour la gestion durable de la diversité biologique dans le cadre de la CDB dont le Cameroun est Partie. Aussi l'approche par écosystème a été proposée comme une stratégie de gestion intégrée des terres, des eaux et des ressources biologiques pour favoriser la conservation et l'utilisation durable de ces ressources d'une manière équitable.

L'étude phytogéographique du Cameroun (Letouzey, 1968) présente le milieu camerounais avec les caractéristiques orohydrographique, géologique et pédologique, climatologique, biodynamiques comportant les facteurs anthropiques d'une part; et d'autre part la végétation avec documents floristiques et vue d'ensemble phytogéographique. Il apparaît déjà dans ce travail la prise en compte de toutes les composantes aussi bien physiques que biologiques d'un écosystème. C'est ce travail préalable qui a servi à l'élaboration de la carte phytogéographique du Cameroun à l'échelle
$1 / 500.000^{\mathrm{e}}$ (Letouzey, 1985) accompagnée de cinq notices qui décrivent les principaux groupements végétaux avec leurs paysage et flore caractéristique, discute du dynamisme de cette végétation tant sur les plans paléobotaniques qu'actuels en tenant compte des influences des activités anthropiques. Elle est accompagnée de cartes spécifiques portant sur le relief, la topographie, l'hydrographie, les sols, le climat. Letouzey $(1968,1985)$ constituent donc une base scientifique robuste et un modèle appropriés pour l'objectif de ce travail qui est de proposer une carte des écosystèmes du Cameroun conforme à la nomenclature de la CDB. Cette carte servira d'outil de planification et de décision pour la gestion durable des ressources biologiques, de matériel dicdactique pour la biologie de la conservation en ce qui concerne les écosystèmes et enfin de recherche pour les sciences environnementales.

\section{MATÉRIEL ET MÉTHODES Matériel}

Deux cartes ont été utilisées pour établir la carte des écosystèmes du Cameroun : 1) la carte phytogéographique du Cameroun (Letouzey, 1985) dont les huit feuillets originaux ont été scannés et assemblées, et la carte du relief et hydrographie (Tchawa, 2006). La superposition des deux cartes met ensemble les données physiques et les phytochories du Cameroun.

\section{Méthodes}

La méthode est d'utiliser les données déjà publiées (Witté et Touroult, 2014 ; David et al., 2001). Puis l'analyse de la nomenclature de la $\mathrm{CDB}$, la délimitation des écosystèmes et le calcul des superficies permettront de cartographier les écosystèmes.

\section{Référentiels bibliographiques}

Les caractères écologiques ayant servi de critères pour la nomenclature, présentés dans les documents ci-dessous, ont été exploités pour délimiter les écosystèmes du Cameroun :

1) pour les caractères physiques:

a) les climats : Morin (1979a, 1979b), Tsalefack (2006); 
b) le relief: Ben Yamed et al. (2006); Ngako (2006) ; Tchawa (2006);

c) l'hydrographie : Morin (1979) ;

2) pour les caractères biologiques, la végétation actuelle: Achoundong (2006); Bikié et al. (2000) ; Guillaumet et al. (2009); World Ressources Institute, USAID-CARPE (2008); Letouzey $(1968,1985)$; White (1979).

\section{Analyse de la nomenclature des écosystèmes}

Les écosystèmes du Cameroun sont cartographiés par l'analyse de la nomenclature des écosystèmes selon la Conventions sur la Diverstié Biologique (CDB). Il est admis que le territoire du Cameroun abrite six écosystèmes (Republic of Cameroon, 2009 ; République du Cameroun, 2014). Les caractéristiques de la nomenclature sont présentées ainsi qu'il suit du nord vers le sud, et de l'ouest vers l'est :

1) l'écosystème d'eau douce: le réseau hydrographique des eaux intérieures est le critère de l'écosystème d'eau douce. Les rivières et lacs forment un réseau hydrographique continental généralement réparti en bassins versants. Le bassin versant considéré comme un " système " est une surface élémentaire hydrologiquement close, c'est-à-dire qu'aucun écoulement n'y pénètre de l'extérieur et que tous les excédents de précipitations s'évaporent ou s'écoulent par une seule section à l'exutoire.

Le réseau des cours d'eau est étroitement lié au relief. En effet, les rivières naissent dans les terres en hauteur (altitude moyenne entre 700 et $1200 \mathrm{~m}$ ) et coulent dans les vallées ou parties basses des terres émergées. Ainsi les grands cours d'eau du Cameroun prennent, pour la plupart, naissance dans les massifs de ligne volcanique du Cameroun dont les Monts Mandara pour le Mayo Louti, le Mayo Tsanaga ou le Mayo Zouvou qui rejoignent plus bas le Mayo Kebi un affluent nord de la Bénoué ; le plateau de l'Adamaoua pour la Sanaga, le Faro, la Bénoué ou le Mayo Rey; les hautes terres de l'Ouest pour le Noun, le Mbam; ou les montagne du sud-ouest avec le Mungo ou le Wouri qui coulent vers l'Océan Atlantique ; et le plateau central pour le Nyong, ou des affluents du Ntem qui coulent vers l'Océan atlantique à l'ouest et le Congo vers l'Est pour le Dja et la Boumba. Il faut noter le système intérieur de la vallée du Logone qui coule vers le Lac Tchad.

Les phytochories sont les forêts marécageuses, rupicoles ou périodiquement inondées, et les prairies marécageuses ;

2) l'écosystème semi-aride: la non disponibilité de l'eau du sol est caractérisée par le degré d'aridité qui est le caractère principal de cet écosystème. L'aridité est un phénomène climatique impliquant une pluviométrie faible. On retrouve trois degrés communs à quasiment tous les indices d'aridité selon les auteurs. Conformément à cet indice, la zone semi-aride a un indice d'aridité compris entre 0,20 et 0,50 . Le climat semi-aride est ainsi défini comme la zone dans laquelle les précipitations sont, certaines années, insuffisantes pour y maintenir les cultures et où l'évaporation excède souvent les précipitations. Il s'agit donc d'une zone subdésertique située entre le sahel (au-dessus de la latitude $13^{\circ} \mathrm{N}$ et la zone soudanienne), caractérisée par une saison sèche s'étendant sur la plus grande partie de l'année et une saison "humide», avec de faibles précipitations (pluviométrie comprise entre 200 et $400 \mathrm{~mm} / \mathrm{an}$ ).

$\mathrm{Au}$ Cameroun, la zone semi-aride couvre la partie du territoire soumise au domaine des climats tropicaux, climat tropical soudano-sahélien (Tsalefack, 2006) au nord entre $10^{\circ}-13^{\circ} \mathrm{N}$ avec de décembre à avril, 7 mois et plus secs, peu de pluies pendant 3 mois en juillet-août -septembre quand la mousson atteint cette région (pluviométrie annuelle de $810 \mathrm{~mm}$ à Maroua) et 1 mois moins humide en septembre-octobre pendant la période de non influence de la mousson. Les températures sont élevées (Kousseri $35^{\circ} \mathrm{C}$ ) à cause de la forte insolation due à l'absence ou une faible présence de nuages pendant la majeure partie de l'année.

3) l'écosystème de savane tropicale boisée: la végétation de savane tropicale est le principal caractère écologique de cet écosystème.

Le terme savane a été utilisé pour la première fois en Amérique du sud pour désigner les plaines septentrionales et des Antilles couvertes d'herbes plus ou moins xérophiles et de buissons avec quelques arbres ou arbustes (Adjanohoun 1964). L'appellation 
a, par la suite, été étendue à la végétation d'Afrique tropicale qui comporte de nombreuses similitudes avec celle de l'Amérique centrale. Selon Aubréville (1957) et Descoings $(1973,1978)$ le terme savane est définie comme une formation végétale propre aux régions chaudes à longue saison sèche et dominée par les plantes herbacées de la famille botanique des Poaceae (ou Graminae), plus ou moins parsemée d'arbres ou d'arbustes. C'est donc une formation herbeuse par définition, la présence de l'élément ligneux est signalée par le qualificatif "boisée". Savane boisé est donc une formation herbacée avec la présence de ligneux qui peuvent être de petits arbres, des arbustes, ou des arbrisseaux rabougris en une seule strate (savane arbustive); ou alors de petits arbres disséminés avec la présence d'arbustes, donc deux strates (savane arborée). Le terme «tropical» renvoie aux climats tropicaux (Tsaléfac 2006). L'écosystème de savane tropicale boisée au Cameroun englobe alors sur le plan géographique tout le territoire du domaine des climats tropicaux et la partie nord du domaine des climats subéquatoriaux et équatoriaux. Les phytochories rencontrées sont les savanes arbustives soudanosahéliennes (climat tropical soudanien de cuvette), arbustives et arborées médiosoudaniennes, voire des forêts claires (à ne pas confondre avec les forêts sèches qu'on rencontre en Australie) soudano-guinéennes (climat tropical humide d'altitude); les savanes péri-forestières ou arbustives guinéosoudaniennes (climat subéquatorial) ;

4) l'écosystème de montagne : le relief est le principal caractère physique du milieu de cet écosystème. Une montagne est une élévation importante et naturelle du sol caractérisée par une forte dénivellation entre sommets et fonds de vallées. Le relief est le résultat des mouvements tectoniques qui se déroulent au cours de l'histoire d'un continent. Ainsi l'histoire géologique du Cameroun est faite des grands mouvements tectoniques du socle africain qui se sont déroulés durant les dernières quatre milliards d'années (Ben Yamed, 2006; Ngako, 2006).

D'après ces auteurs, il y a eu formation durant deux premiers cycles orogéniques non volcaniques (étant essentiellement dus à des plissements qui ont provoqués des bombements du socle cristallin) des monts de moyenne hauteur, comme le massif de Ngovayang ou des collines autour d'Ebolowa pour le complexe du Ntem, des Mamelles vers Kribi pour la série du Nyong, des inselbergs pour la série d'Ayna, des massifs autour de Yaoundé tels que Eloumden, Kala et Mbamimkom, de Matomb au centre; et à l'est les Monts Guimbiri près d'Abong Mbang pour le groupe de Yaoundé.

Puis s'est produit le cycle orogénique Panafricain qui a d'une part provoqué la formation des failles structurantes de direction SW-NE, avec l'effondrement du socle; et d'autre part le volcanisme avec l'infiltration et l'émergence en surface du magma qui est remonté par les fractures et a bâti les hautes terres (altitude jusqu'à $4000 \mathrm{~m}$ de hauteur) formant la ligne volcanique du Cameroun ou dorsale camerounaise. Les montagnes sont réparties ainsi qu'il suit :

- à l'extrême nord les Monts Mandara (900 m d'altitude) avec des pics au nord de Mokolo (1442 m) et au sud de Mogode (1224 m) qui sont sur le prolongent septentrional de la ligne volcanique du Cameroun. Ces collines bien que n'atteignant que l'étage submontagnard font bien partie de cet écosystème à cause de leur flore où on trouve des espèces végétales véritablement montagnardes dont certaines apparentées à celles des hautes montagnes de l'Afrique orientale et d'Abyssinie en particulier, sans qu'elles existent sur les autres montagnes camerounaises plus au sud (Letouzey, 1985) ;

- au nord les Monts Alantika (1885 m) dont le sommet se trouve au Nigeria voisin et les Monts de Poli avec l'Hosséré Vokré (2049 m) ;

- sur le plateau de l'Adamaoua, les sommets granitoïdes ou volcaniques ici sont des "Hosséré", ou des massifs surrélévés appelés "Tchabal" avec les plus hauts sommets à l'Ouest dont Tchabal Ouadé $(2418 \mathrm{~m})$, Tchabal Mbabo (2460 m), Tchabal Ngangdaba (1960 m) et Tchabal Ngangha (1923 m); et vers l'Est des pointements dont les Monts Wal ou la Montagne de la Ngaï éparpillés çà et là ;

- à l'ouest les hautes terres de l'Ouest constitués d'une succession de massifs 
montagneux d'altitude 1500 à $3000 \mathrm{~m}$ dont le Montt Oku (3008 m), les Monts Bamboutos $(2740 \mathrm{~m})$, les Monts Manengouba (2396 m), le Mont Koupé $2050 \mathrm{~m}$ ou les Monts Rumpi (1764 m);

- au sud-ouest, sur la plaine littorale dont le relief plat est brusquement surélevé au centre par la dorsale camerounaise qui forme sur la côte le massif isolé du Mont Cameroun qui culmine à $4070 \mathrm{~m}$.

L'écosystème de montagne est donc un archipel de massifs montagneux d'au moins $800-1200 \mathrm{~m}$ au nord à 1800-2000 m d'altitude au sud et comporte les étages submontagnards et montagnards où la végétation est constituée de forêts qui poussent à des altitudes comprises entre 1.000 et $3000 \mathrm{~m}$; et l'étage subalpin $(3000-4000 \mathrm{~m}$ d'altitude) qui est couvert de prairies subalpines, de mousses et lichens sur les rochers;

5) l'écosystème de forêt tropicale dense humide: La forêt dense humide est le principal caractère écologique de cet écosystème. $\mathrm{La}$ végétation est entendue comme l'ensemble des groupements végétaux d'une aire géographique. Elle est caractérisée par sa physionomie et sa structure. Ces caractéristiques sont étroitement liées aux caractères physiques du milieu dont le climat en est le principal. A l'observation, il apparaît d'ailleurs une correspondance forte entre les limites des types de climat (Tsalefack, 2006) et celles des types de végétation (Achoundong, 2006; Letouzey, 1985).

Il existe beaucoup de définitions du terme forêt (Arnould, 2001). Aussi le mot "forêt" s'applique à des formations excessivement variées de toutes les zones climatiques (Aubréville, 1965) en tenant compte du contexte biogéographique. Par exemple au Sahel, un boisement est considéré comme forêt à partir d'un taux de recouvrement de 10\%, alors qu'en Europe, on ne parle de forêt qu'à partir d'un taux de recouvrement de $20 \%$ et d'une surface de plus d'un demi-hectare. Selon l'Organisation des Nations Unies pour l'Agriculture et l'Alimentation (Food and Agriculture Organization of the United Nations, 1998), la forêt est définie comme des terres occupant une superficie de plus de 0,5 ha avec des arbres atteignant une hauteur supérieure à cinq mètres et un couvert arboré de plus de $10 \%$, ou avec des arbres capables d'atteindre ces seuils in situ. C'est cette dernière définition qui est la plus utilisée par les administrations et autres institutions. L'arbre est défini ici comme une plante pérenne avec une seule tige (ou plusieurs si elle est recépée) atteignant au moins cinq mètres à maturité. Cette définition exclut les terres dont la vocation prédominante est agricole ou urbaine. Quant à la superficie 0,5 ha, elle semble liée au concept écologique de l'aire minimale en zone forestière qui est "une surface suffisamment grande pour contenir la quasi-totalité des espèces présentes sur l'individu d'association" (Guinochet, 1973). En effet, Letouzey (1968) indique qu'en forêt tropicale dense humide au Cameroun, une aire de 0,5 ha est suffisante pour contenir l'aire minimale quel que soit le type de forêt. Des définitions plus spécifiques ont été énoncées par d'autres institutions dont les deux exemples suivants :

- le Programme des Nations unies pour l'environnement (PNUE) utilise $40 \%$ de couverture comme le seuil pour les «forêts fermées » et 10 à $40 \%$ de couverture pour les «forêts ouvertes » (Food and Agriculture Organization of the United Nations, 1998),

- le projet Tropical Ecosystem Environment Observations by Satellite (TREES), fondé en 1991 par la Commission Européenne, classifie les surfaces avec plus de $70 \%$ de couverture de canopée comme étant des «forêts denses » et celles avec 40-70\% de couverture comme des «forêts fragmentées » (Tchatchou et al., 2015).

Du point de vue de l'écologie, la forêt est un écosystème complexe et riche, offrant des habitats à de nombreuses espèces et populations animales, végétales, fongiques et microbiennes entretenant entre elles, pour la plupart, des relations d'interdépendance.

Selon Aubréville (1965), la définition des formations végétales, leur classification et leur nomenclature peuvent être considérées d'après quatre ordres de considération : physionomiques, écologiques, floristiques et évolutives (ou syngénétiques c'est-à-dire liés au dynamisme du groupement végétal bien souvent dû aux activités anthropiques). 
La désignation "forêt tropicale dense humide" se réfère à la forêt comme type de végétation dominante; le climat est de type tropical avec alternance d'une ou de deux saisons de pluies et d'une ou de deux saisons sèches respectivement; le couvert végétal fermé qui implique la densité élevée d'arbres et l'humidité est due à une pluviométrie élevée au moins égale à $1500 \mathrm{~mm}$ par an. Dans cette nomenclature, il n'est pas tenu compte de la considération évolutive (forêt primaire, mature, secondaire, fragmentée, dégradée ou en cours de reconstitution);

6) l'écosystème marin et côtier: le caractère écologique de l'écosystème marin et côtier est l'hydrographie dans sa composante de l'eau salée de la mer et l'eau saumâtre des mangroves.

La mer est l'ensemble des eaux océaniques, communiquant entre elles et ayant le même niveau de base. C'est aussi une division de l'océan mondial définie du point de vue hydrographique (limites continentales ou insulaires) et hydrologique (température, salinité, courants) (Dictionnaire de français Larousse). Le terme générique "mer" peut aussi désigner une grande étendue d'eau salée différente des océans, l'ensemble des espaces d'eau salée en communication libre et naturelle sur toute l'étendue du globe ou encore une grande étendue sombre à la surface de la Lune. Le terme "mer" est aussi utilisé pour désigner certains grands lacs, en particulier lorsqu'ils n'ont pas de cours d'eau dans lesquels ils se déversent (Wikipedia, 2017).

Le littoral est la zone sinueuse où s'établit le contact entre la mer ou un lac et la terre ferme. C'est une bande de terre constituant la zone comprise entre une étendue maritime et la terre ferme, le continent, ou l'arrière-pays. Selon les échelles retenues, le littoral peut s'étendre de quelques centaines de mètres à plusieurs kilomètres de part et d'autre de la limite terre-eau ou au sens strict, correspondre à l'estran qui est la zone de balancement des vagues ou la côte. Il est typiquement constitué de l'étage infralittoral ou plateau continental qui est la partie marine, et l'étage supra-littoral qui est la terre ferme ou rivage.
Sous les eaux marines, le plateau continental constitue la partie immergée du littoral. Il comprend la partie située sous la mer intérieure (12 miles) et la zone contiguë (12 miles) ou jusqu'à 200 milles marins des lignes de base, lorsque ce rebord externe se trouve à une distance inférieure. Sa limite coïncide alors avec celle de l'extension maximale de la zone économique exclusive (ZEE). Comme la ZEE, sa limite peut, toutefois, se situer à moins de 200 milles dans le cas où les côtes de deux États sont adjacentes ou se font face (cas entre le Cameroun et la Guinée Equatoriale, île Bioko). Le Cameroun est ouvert à l'Océan Atlantique au fond du Golfe de Guinée sur une longueur de $420 \mathrm{~km}$, le relief du rivage et de la côte est une plaine d'altitude 0 à $300 \mathrm{~m}$ en arc de cercle autour du golfe de Guinée sur une largeur d'environ 100 à $200 \mathrm{~km}$. Mais la côte concerne une bande environ $50 \mathrm{~km}$ sur sol jaune hydromorphe.

La végétation de l'écosystème marin et côtier est constituée de phytoplancton dans l'eau, d'algues marines, de mangroves sur la côte et forêts sur cordons littoraux du rivage.

\section{Délimitation des écosystèmes}

La délimitation d'unités écosystémiques tient d'abord compte des phytochories ou unités de végétation au niveau des secteurs phytogéographiques (Letouzey, 1985). C'est pourquoi il a été nécessaire d'établir une carte de végétation «simplifiée » qui ne tienne compte que des secteurs phytogéographiques. Cette délimitation se réfère à celles des royaumes biogéographiques (biogeographical-realms), et des biomes qui sont basées sur les divisions floristiques (David et al., 2001) bien que les critères tiennent compte de toutes les composantes de la diversité biologique (Morrone, 2015).

Calcul des superficies occupées par les écosystèmes. La carte des écosystèmes a été quadrillée par unités d'un degré carré. C'est une méthode pour quantifier la biodiversité par unité de surface (Chirio et LeBreton, 2007 ; Onana, 2011). Au niveau de l'équateur, la distance entre deux parallèles d'un degré de longitude est estimée à environ $111 \mathrm{~km}$, souvent on ramène à environ $100 \mathrm{~km}$; tandis que pour la latitude la distance entre deux 
méridiens est de $11 \mathrm{~km}$. Afin de rendre plus facile une évaluation des distances et donc du calcul d'une superficie d'une aire donnée, on utilise une représentation plane ou projection de la terre (IGN, 2008). Dans notre cas, nous considérons les cartes planes IGN au $1 / 200.000^{\mathrm{e}}$ du Cameroun dont le côté correspond à une distance de $111 \mathrm{~km}$ entre deux parallèles, soit une aire d'environ $12.321 \mathrm{~km}^{2}$ par degré carré. Etant donné que certaines unités sont des limites d'au moins deux écosystèmes, et d'autres se trouvent à cheval sur un pays voisin du Cameroun (Figure 3), un pourcentage d'occupation de chaque écosystème a été appliqué afin d'en évaluer la superficie correspondante.

\section{RÉSULTATS}

La carte de la végétation a été élaborée (Figure 1). Puis la superposition de la carte hydrographique et des couches des cartes du relief sur celle de la végétation a permis d'établir la délimitation spatiale et la superficie approximative des écosystèmes du nord au sud et de l'ouest à l'est (Tableaux 1, 2 ; Figures 2,3).

\section{Écosystème d'eau douce}

Il couvre la partie camerounaise du Lac Tchad, la vallée inondable du Logone, les rivières, fleuves et zones inondables induites, lacs, marécages permanents ou périodiques. Les cartes IGN au $1 / 200.000^{\mathrm{e}}$ sont celles de l'ensemble du territoire parfois sur moins de $1 \%$, si bien que la superficie totale occupée est difficile à estimer. Cependant, il a été estimé à un peu plus de $7800 \mathrm{~km}^{2}$ la superficie occupée par les lacs naturels ou artificiels, les marécages et zones inondables.

\section{Écosystème semi-aride}

Il couvre les abords du Lac Tchad, la plaine du Diamaré et en partie la cuvette de la Bénoué. Les cartes IGN 1/200.000e sont celles de Makary, Mora, Mokolo, Maroua, Garoua (et Léré), Yagoua (Bongor) sur une superficie d'environ $25.000 \mathrm{~km}^{2}$.

\section{Écosystème de savane tropicale boisée}

Il couvre une partie de la cuvette de la Bénoué, la plaine du Nord, le plateau de l'Adamaoua et les hautes terres de l'Ouest. Il est réparti sur les cartes IGN $1 / 200.000^{\mathrm{e}}$ qui forme le polygone Mokolo-Mamfe-BafiaBatouri-Mokolo sur une superficie d'environ $166.000 . \mathrm{km}^{2}$.

\section{Ecosystème de montagne}

Il est réparti sur un archipel sur les montagnes d'au moins 1500-1800 m d'altitude sur la ligne volcanique ou dorsale du Cameroun. Les cartes IGN $1 / 200.000^{\mathrm{e}}$ sont celles de Mokolo, Tignère, Baléké-Mbéré, Akwaya, Nkambe, Mamfe, Bafoussam, BueaDouala. C'est le plus petit des écosystèmes car iI couvre une superficie totale d'environ $15.400 \mathrm{~km}^{2}$.

\section{Ecosystème de forêt tropicale dense humide}

Cet écosystème couvre l'ensemble du plateau méridional à l'exception du rebord sud du plateau de l'Adamaoua (occupé par des savanes péri-forestières) jusqu'à la frontière sud du territoire sur toute la largeur (Guillaumet et al., 2009). C'est le plus grand écosystème car il couvre près de la moitié du territoire en superficie (47\%). Il est réparti sur les cartes IGN $1 / 200.000^{\mathrm{e}} \mathrm{du}$ polygone Nkambe-Buea-Douala-Kribi-Moloundou-

Batouri-Bertoua-Deng Deng-LinteNdikinimeki-Mamfe-Nkambe. La superficie est estimée à environ $226.000 \quad \mathrm{~km}^{2}$. Cependant, c'est dans cet écosystème que se trouvent les plus grandes agglomérations, les plus grandes exploitations des ressources naturelles, si bien que l'écosystème est fragmenté et la superficie actuelle des forêts est plus réduite.

\section{Ecosystème marin et côtier}

Il est localisé sur la partie camerounaise du golfe de Guinée sur les cartes IGN $1 / 200.000^{\mathrm{e}}$ de Buea-Douala et Kribi. Il couvre une superficie d'environ $18.000 \mathrm{~km}^{2}$ dont $2.494 \mathrm{~km}^{2}$ de mangrove (Beentje et Bandeira, 2007). 


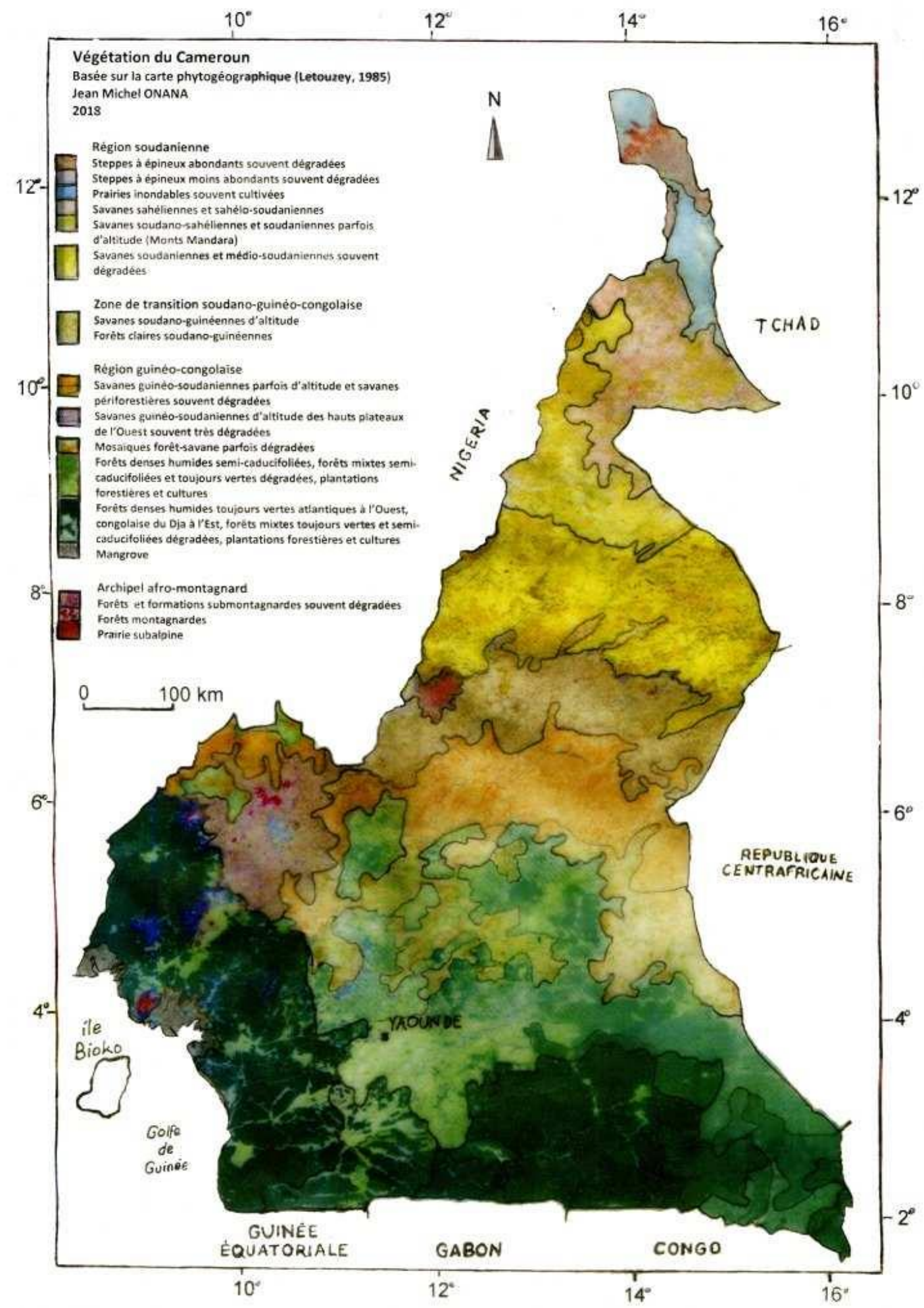

Figure 1: Carte de la végétation du Cameroun. 
Tableau 1: Répartitions spatiale et administrative des écosystèmes du Cameroun.

\begin{tabular}{|c|c|c|}
\hline Ecosystème & Répartition spatiale & $\begin{array}{l}\text { Répartition } \\
\text { administrative } \\
\text { (Régions) }\end{array}$ \\
\hline Eau douce & $\begin{array}{l}\text { rivières, fleuves, lacs, zones continentales } \\
\text { humides (marécages, zones inondables) }\end{array}$ & Toutes les Régions \\
\hline Semi-aride & plaine du Diamaré, cuvette de la Bénoué & Extrême-Nord, Nord \\
\hline $\begin{array}{l}\text { Savane } \\
\text { tropicale } \\
\text { boisée }\end{array}$ & $\begin{array}{l}\text { cuvette de la Bénoué, plateau de l'Adamaoua, Grass } \\
\text { et plateau Bamiléké (moins de } 800-1200 \mathrm{~m} \\
\text { d'altitude) ; nord et nord-est du plateau sud- } \\
\text { camerounais }\end{array}$ & $\begin{array}{l}\text { Nord, Adamaoua, Nord- } \\
\text { Ouest, Ouest, Centre, Est }\end{array}$ \\
\hline Montagne & $\begin{array}{l}\text { Dorsale camerounaise ou Ligne volcanique du } \\
\text { Cameroun (altitude supérieure à 1200m à l'extrême } \\
\text { nord) : Monts Mandara ; (altitude supérieure à } \\
1800-2000 \text { m ailleurs) : Monts de Poli (Hosséré } \\
\text { Vokré), Tchabal Ouadé, Tchabal Mbabo, Tchabal } \\
\text { Ngangdaba, Tchabal Ngangha, Monts Bamboutos, } \\
\text { Monts du Mbam ; Monts Oku ; Monts de Bamenda; } \\
\text { Monts des Bakossi, Monts Rumpi, Monts } \\
\text { Manengouba, Mont Koupé, Mont Nlonako, Mont } \\
\text { Cameroun. }\end{array}$ & $\begin{array}{l}\text { Extrême nord, Nord, } \\
\text { Adamaoua, Nord-Ouest, } \\
\text { Ouest, Sud-Ouest, Littoral }\end{array}$ \\
\hline $\begin{array}{l}\text { Marin et } \\
\text { côtier }\end{array}$ & Plateau continental du golfe de guinée ; mangrove & Sud-Ouest, Littoral, Sud \\
\hline $\begin{array}{l}\text { Forêt } \\
\text { tropicale } \\
\text { dense humide }\end{array}$ & $\begin{array}{l}\text { Plaine littorale, plateau sud-camerounais, plaine de } \\
\text { l'est }\end{array}$ & $\begin{array}{l}\text { Sud-Ouest, Littoral, Centre, } \\
\text { Sud, Est }\end{array}$ \\
\hline
\end{tabular}

Tableau 2: Superficies des écosystèmes et taux d'occupation par rapport à la superficie totale du territoire. Les superficies des écosystèmes ont été arrondies par excès à la centaine supérieure. La superficie du territoire a été arrondie à $476.000 \mathrm{~km}^{2}$ en prenant en compte le plateau continental.

\begin{tabular}{lcc}
\hline Ecosystème & $\begin{array}{c}\text { Superficie approximative } \\
\left(\mathbf{k m}^{\mathbf{2}}\right)\end{array}$ & $\begin{array}{c}\text { Pourcentage par } \\
\text { rapport à la superficie } \\
\text { totale approximative du } \\
\text { territoire }(\%)\end{array}$ \\
\hline Eau douce & 7.800 & 1,64 \\
Semi-aride & 25.000 & 5,25 \\
Savane tropicale boisée & 166.000 & 34,87 \\
Montagne & 15.500 & 3,26 \\
Forêt tropicale dense humide & 226.000 & 47,48 \\
Marin et côtier & 18.000 & 3,78 \\
Total & 458.300 & 96,28 \\
\hline
\end{tabular}




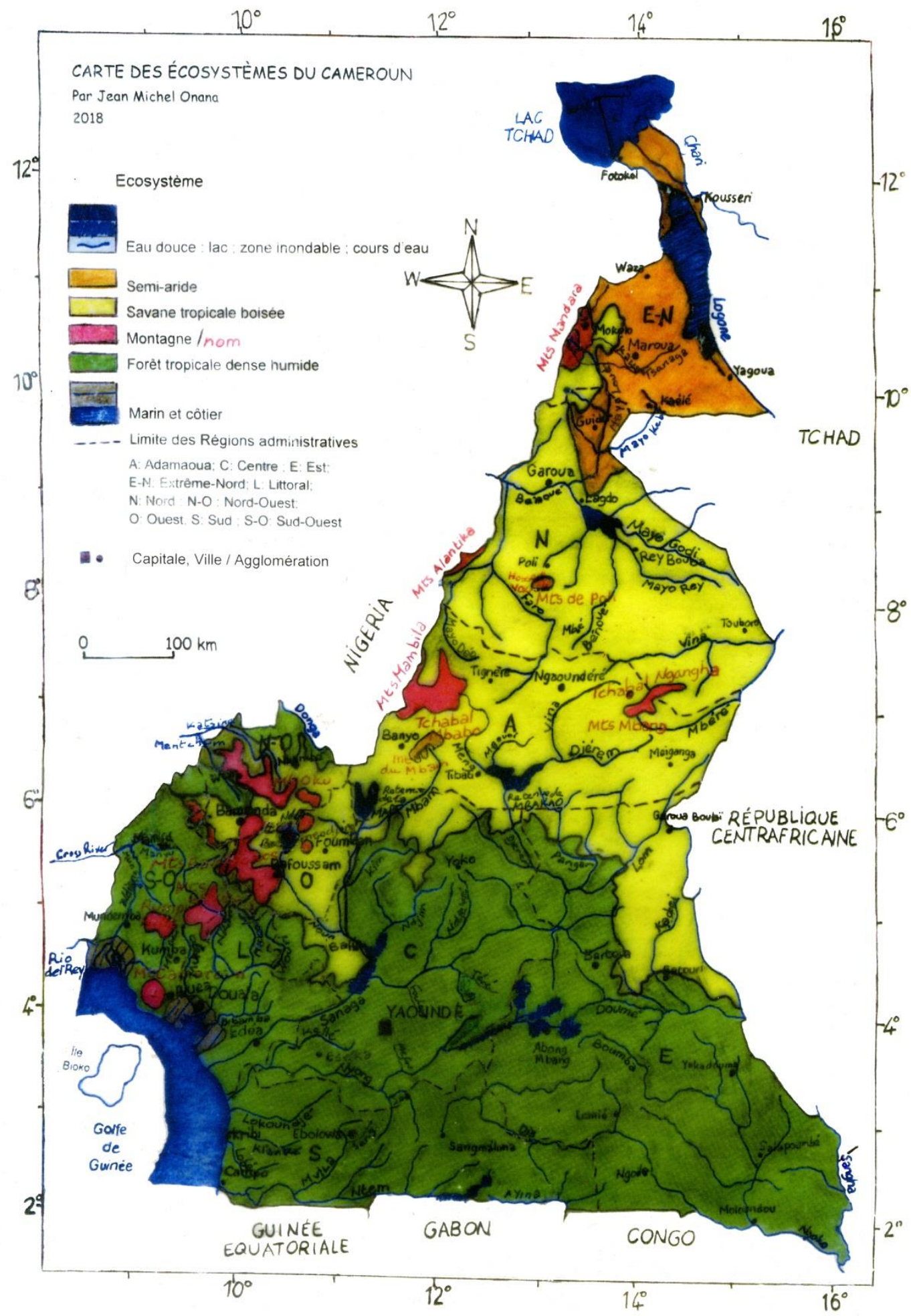

Figure 2: Carte des écosystèmes du Cameroun. 


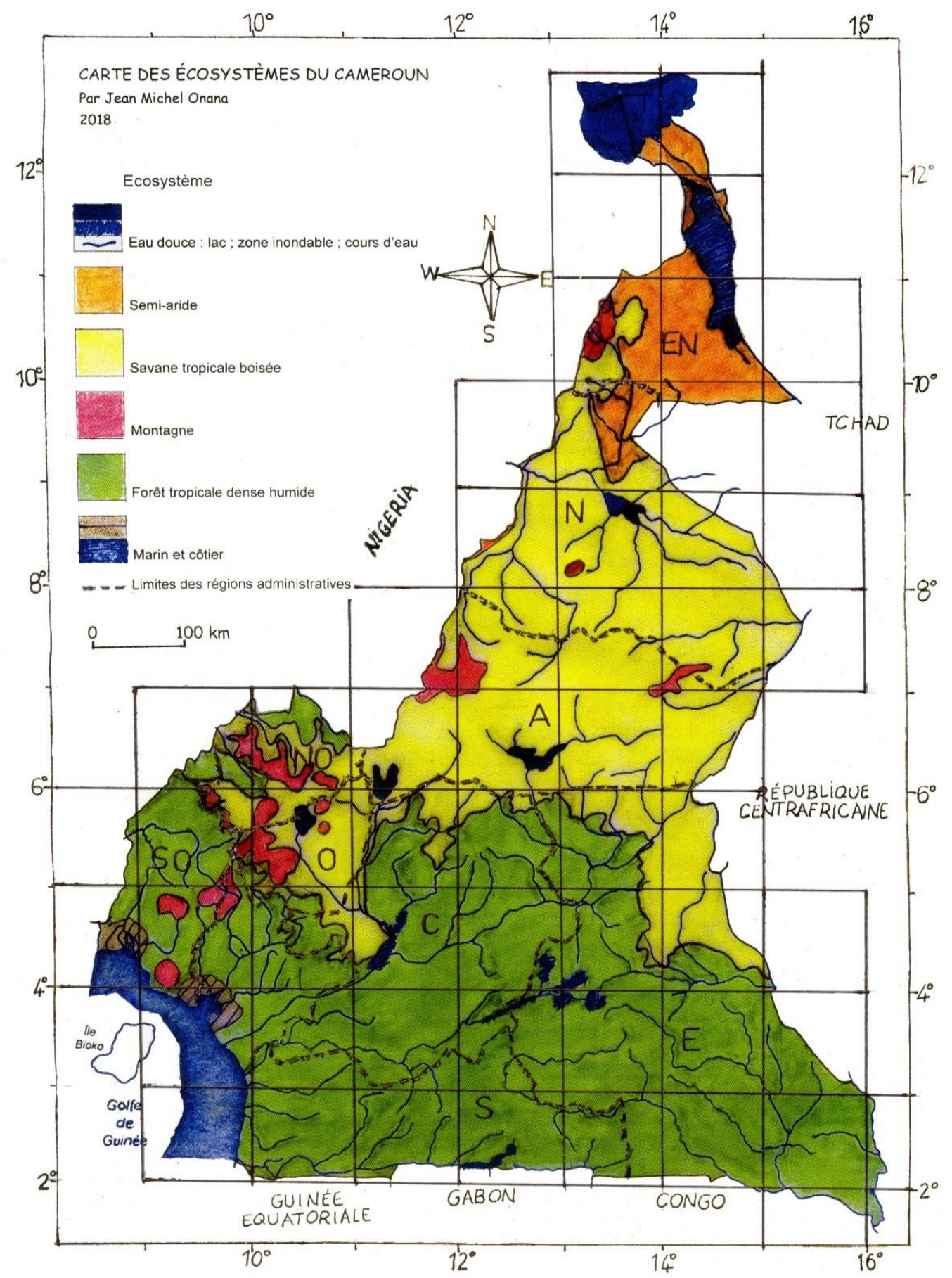

Figure 3: Aperçu de l'occupation des écosystèmes par région administrative et par unités géographiques d'un degré carré. Les régions administratives : $\mathrm{A}=$ Adamaoua, $\mathrm{C}=$ Centre, $\mathrm{E}=$ Est, EN = Extrême-Nord, L = Littoral, $\mathrm{N}=$ Nord, $\mathrm{NO}=$ Nord-Ouest, $\mathrm{O}=$ Ouest $, \mathrm{S}=\mathrm{Sud}, \mathrm{SO}=$ Sud-Ouest ; pour les gazzetiers des cartes IGN. 1/200.000' (Annexe en Afrique de l'I.G.N. 1964, voir couverture des pochettes des cartes ; Onana 2013, p. 35). 


\section{DISCUSSION}

La délimitation des écosystèmes reste approximative à cause principalement de l'interpénétration des groupements végétaux (forêts mixtes, savanes péri-forestières ou contacts forêt-savane) et le caractère continue de la variation des caractères écologiques dont principalement le climat. Aussi la présence de savanes relictuelles incluses dans l'écosystème de forêt (cas des savanes d'Akonolinga et d'Ayos ou au pied du mont Cameroun sur la pente nord selon Letouzey (1985) pose le problème des écosystèmes en bloc homogène; de même les avancées de la forêt qui colonisent les savanes périforestières (cas à Batouri) induisent la différence dans l'étendue de la zone forestière, soit qu'on considère la couverture aérienne ou les groupements phytogéographiques.

La superficie totale des écosystèmes est estimée à $458.300 \mathrm{~km}^{2}$ soit un écart d'environ $17.700 \mathrm{~km}^{2}$ (environ $3,71 \%$ ) par rapport à la superficie totale d'environ $476.000 \mathrm{~km}^{2}$ en prenant en compte le plateau continental. En dehors de la superficie couverte par le domaine forestier dont l'estimation des superficies varie selon les auteurs, soit entre 19.600 et $22.800 \mathrm{~km}^{2}$ pour la décennie 90 (Bikié et al. 2000), 21.200$22.000 \mathrm{~km}^{2}$ (Gonmadje et al. 2012; Unité Technique du Projet d'Inventaire forestier national, FAO 2006) ou $285.000 \mathrm{~km}^{2}$ (environ $60 \%$ du territoire national) d'après l'Atlas Forestier Interactif du Cameroun (Cameroon Ministry of Forestry and Fauna, World Resources Institute, 2007) qui est un système d'information mise à jour en continu et combinant l'utilisation de la télédétection, les Systèmes d'Information Géographiques (SIG) et les vérifications de terrain pour le suivi et la gestion des forêts (Tessa, 2012). Ces chiffres qui correspondent à l'aire de l'écosystème de forêt tropicale dense humide corroborent le chiffre d'environ $230.000 \mathrm{~km}^{2}$ obtenu dans le cadre de ce travail. Cependant, la superficie obtenue à partir de la carte de la couverture forestière (Cameroon Ministry of Forestry and Fauna, World Resources Institute, 2007) semble élevée, car les mosaiques forêts-savanes sont considérées comme des forêts. Les superficies des autres écosystèmes ont été estimées pour la première fois dans ce travail.

Comme on peut le voir sur les cartes de la végétation (Cameroon Ministry of Forestry and Fauna, World Resources Institute, 2007 ; Letouzey, 1985), les écosystèmes de forêt tropicale dense humide, de savane tropicale boisée et semi-aride sont fortement dégradés, si bien que les formations naturelles laissent la place aux mosaïques forêt-cultures, savanecultures ou formations herbeuses-cultures. De même avec l'urbanisation, ces écosystèmes sont fragmentés et complètement modifiés par l'installation d'agglomérations qui constituent ainsi des écosystèmes urbains (Duvigneaud, 1970) inclus dans les écosystèmes naturels.

Par ailleurs, à cause du changement climatique et des activités anthropiques, les caractéristiques des écosystèmes sont dynamiques (Tchouto, 2004; van Germerden, 2003 ; Maley, 1987, 1990, 1994, 2001; Mittermeier et al., 2004; Momo 2009; Momo et al., 2012; Neumann et al., 2012). Par exemple, au nord Cameroun, à cause du phénomène de la désertification, on pourrait passer de l'écosystème de zone semiaride à l'écosystème du désert du fait de l'aggravation de l'aridité (Ntoupka, 1999). De même, les paysages forestiers de l'écosystème de forêt tropicale dense humide subissent de profondes modifications du fait des activités humaines (Ngomanda et al., 2009), en particulier l'urbanisation et de ce fait les zones urbanisées deviennes des hotspots de biodiversité (Onana et Cheek, 2011). De ce fait, la connaissance de la distribution des écosystèmes sera un instrument pour la gestion durable selon d'autres concepts tels que Tropical Important Plant Areas (Darbyshire et al., 2017) ou Key Biodiversity Areas (IUCN, 2016). Aussi les services écosystémiques seront mieux identifiés par écosystème et plus efficacement gérés, alors que jusqu'ici, les différents travaux et rapports ne portent que sur la forêt bien que la définition adoptée incluait aussi les savanes boisées (Gonmadje, 2012; Ministère de l'Environnement et des Forêts et al., 1995 ; 
Unité Technique du Projet d'Inventaire forestier national, FAO, 2006 ; République du Cameroun, 2014).

Plusieurs concepts tels que celui des royaumes ou domaines biogéographiques divisés en biomes puis en écorégions ont été proposés pour la gestion des ressources biologiques dans le monde en harmonie avec les activités humaines afin de favoriser la conservation (David et al., 2001). Les royaumes biogéographiques sont définis comme étant de grandes régions spatiales au sein desquelles les écosystèmes partagent une histoire biologique évolutive globalement similaire. Huit royaumes biogéographiques terrestres sont typiquement reconnus, correspondant approximativement aux continents. L'Afrique est dans le royaume Afrotropique et on retrouve 10 écorégions au Cameroun (WWF, 2017). Bien que des écosystèmes similaires (tels que les forêts tropicales humides) partagent des processus similaires et des types de végétation importants partout où ils sont trouvés, leur composition en espèces végétales varie considérablement en fonction du domaine biogéographique dans lequel ils se trouvent. L'évaluation de la biodiversité au niveau des domaines biogéographiques est confrontée à différents moteurs de changement, et il peut y avoir des différences dans les options d'atténuation ou de gestion des facteurs. C'est pourquoi il est plus rationnel que la gestion durable des ressources biologiques se fasse par l'approche écosystème et tienne compte des secteurs de gestion des ressources (République du Cameroun, 2014 ; Secrétariat de la Convention sur la Diversité Biologique, 2004). L'approche par écosystème est fondée sur l'application de méthodologies scientifiques adéquates concentrées sur des paliers d'organisation biologique. C'est une excellente stratégie de gestion intégrée à une échelle variable selon la nécessité : ce pourrait être, par exemple, un grain de terre arable, un étang, une forêt ou une espèce emblématique de la faune sauvage (Williams et al., 2000). La cartographie d'unités écosystémiques est ainsi une réponse à une lacune qui permet de visualiser sur la carte du Cameroun, la distribution des écosystèmes tels qu'éditée par la nomenclature de la CDB. C'est un outil essentiel de l'exercice de la souveraineté sur la biodiversité, pour la planification des activités tant de développement que de recherche et de conservation de la diversité biologique à l'échelle nationale (Williams et al., 1997 ; David et al., 2001 ; Republic of Cameroon, 2012; Morrone, 2015).

\section{Conclusion}

Les six écosystèmes: eau douce, semiaride, savane tropicale boisée, montagne, forêt tropicale dense humide, marin et côtier du Cameroun ont été cartographiés. La production de la carte a été réalisée suivant les critères retenus dans la nomenclature des écosystèmes de la CDB, en tenant rigoureusement compte des caractères biophysiques. La carte des écosystèmes vise à combler une lacune dans le dispositif des outils de la gestion durable de la diversité biologique au Cameroun. C'est aussi une contribution à la mise en œuvre du premier engagement de la CBD relatif à l'amélioration des connaissances pour la conservation de la diversité biologique dans toutes ses composantes et ainsi l'exercice de la souveraineté sur les ressources biologiques. Aussi les recherches et les enseignements en biologie de la conservation ou sciences environnementales seront améliorés car il n'y aura plus d'ambiguïté sur le concept d'écosystème et la distribution des écosystèmes au Cameroun. Cependant, étant donné que la méthode de gestion des ressources biologique préconisée est l'approche par écosystème, la carte des écosystèmes est d'abord un outil de planification stratégique au niveau national. C'est pourquoi, dans le prochain article, les caractéristiques des habitats dans les écosystèmes seront présentées afin de permettre une planification ou une exploitation à l'échelle régionale ou locale.

\section{CONFLIT D'INTERETS} d'intérêts.

L'auteur déclare n'avoir aucun conflit 


\section{CONTRIBUTIONS DES AUTEURS}

Cet article a été préparé par le seul présent auteur.

\section{REMERCIEMENTS}

Les remerciements de l'auteur vont à Monsieur Ngansop Eric, étudiant en Master de biologie végétale à la Faculté des Sciences de l'Université de Yaoundé I et cadre scientifique de recherche à l'Herbier national du Cameroun pour la mise en forme des cartes; au Dr Marie Florence Ngo Ngwé, Chef de Station de l'Herbier National pour l'utilisation du matériel technique; à Monsieur Xander van der Burgt de Royal Botanic Gardens, Kew (R.U. de Grande Bretagne) et Monsieur Hervé Chevillotte de l'Institut de Recherche pour le Développement (France) pour leur assistance au cours de la rédaction du manuscrit.

\section{REFERENCES}

Achoundong. 1996. Les Rinorea et l'étude des refuges forestiers en Afrique. In Dynamique à Long Terme des Ecosystèmes Forestiers Intertropicaux, Servant M, Servant-Vildary S (eds). Publications issues du Symposium international "Dynamique à long terme des écosystèmes forestiers intertropicaux, Paris, 20-22 mars 1996 ; 19-29.

Achoundong. 2006. Le Cameroun. Végétation. In Atlas du Cameroun, Collection Atlas de l'Afrique, Ben Yamed D, Houstin N, Seignobos C (eds). Les Editions J.A. Paris ; 64-65.

Adjanohoun E. 1964 Végétation des savanes et des rochers découverts en Côte d'Ivoire Centrale. ORTSOM (IRD).

Amiet J-L. 1987. Aires disjointes et taxons vicariants chez les Anoures du Cameroun: implications paléoclimatiques. Alytes, 6(3-4): 99-115.

Amiet J-L. 2012. Les Rainettes du Cameroun (Amphibiens Anoures). Ed. Jean-Louis Amiet \& la Nef des livres: SaintNazaire; 31-37.

Annexe en Afrique de l'I.G.N. 1964. Cartes de l'Afrique Centrale, République Fédérale du Cameroun au 1/200.000 ${ }^{e} 2^{\mathrm{e}}$ édition. Institut Géographique National: Paris.

Arnould P. 2001. Qu'est-ce qu'une forêt. In Forêts, Lorgnier A (ed). Collection l'Aventure du Monde. Ed. Georges Neaf.: France; 23.

Aubréville A. 1965. Principe d'une systématique des formations végétales tropicales. Adansonia sér., 2(5): 153196.

Beentje H, Bandeira S. 2007. Field guide to the mangrove trees of Africa and Madagascar. Kew Publishing. Royal Botanic Gardens, Kew; 13.

Ben Yamed D, Houstin N, Seignobos C (eds) 2006. Le continent africain. Géologie. In Atlas du Cameroun, Collection Atlas de l'Afrique. Les Editions J.A Paris ; 1213.

Bikié H, Collomb J-G, Djomo L, Minnemeyer S, Ngoufo R, Nguiffo S. 2000. Aperçu de la Situation de l'Exploitation Forestière au Cameroun. Un rapport de l'Observatoire Mondial des Forêts Cameroun. World Resources Institute.

Cameroon Ministry of Forestry and Fauna, World Resources Institute 2007. Vegetation cover in Cameroon. Interactive Atlas of forestry resources of Cameroon, version 2.0.

Chirio L, LeBreton M. 2007. Atlas des reptiles du Cameroun. Publications Scientifiques $d u$ Muséum, 67. IRD Editions, Paris ; 22-24.

Convention on biodiversity 2012 Strategic plan for biodiversity 2011-2020, including Aichi Biodiversity Targets. United Nations; 1-2. https://www.cbd.int/doc/strategic-plan/ 2011-2020/Aichi-Targets-EN.pdf

Convention sur la Diversité Biologique 2012. Stratégie Mondiale pour la Conservation des Plantes : 2011-2020. Botanic Gardens Conservation International, Richmond, UK; 1-36. https://www.bgci.org/files/Plants2020/G SPCbrochure/gspc_french.pdf.

Darbyshire I, Anderson S, Asatryan A, Byfield A, Cheek M, Clubbe C, Ghrabi Z, Harris T, Heatubun CD, Kalema J, Magassouba S, McCarthy B, 
Milliken W, de Montmollin B, Lughadha EN, Onana JM, Saïdou D. Sârbu A, Shrestha K, Radford E. 2017. Important Plant Areas: revised selection criteria for a global approach to plant conservation. Biodivers Conserv. DOI: 10.1007/s10531-017-1336-6

David MO, Dinerstein E, Wikramanayake ED, Burgess ND, Powell GVN, Underwood EC, D'amico JA, Illanga Itoua, Strand HE, Morrison JC, Loucks CJ, Allnutt TF, Ricketts T H, Yumiko Kura, Lamoreux JF, Wettengel WW, Prashant Hedao, Kassem KR (2001). Terrestrial Ecoregions of the World: A New Map of Life on Earth. BioScience, 51(11). Pdf. DOI: https:// academic.oup.com/bioscience/articleabstract/51/11/933/227116;

Descoings B. 1973. Les formations herbeuses africaines et les définitions de Yangambi considérés sous l'angle de la structure de la végétation. Adansonia sér. 2, 13(4): 391-421.

Descoings B. 1978. Les formations herbeuses dans la classification herbeuse de Yangambi Adansonia sér. 2, 18(2) : 243256.

Dictionnaire de français Larousse. www.larousse.fr/dictionnaires/francais/m er/50562. Consulté le 18 Mars 2017 à 17h GMT.

Duvigneaud P, "Ecole de Bruxelles" 1970 Ecosystèmes Urbains. Source : https://bethleemecosysteme. wordpress.com/ecosystemes-urbains/

Food and Agriculture Organization of the United Nations 1998. The state of food and agriculture 1998. FAO Agriculture Series No. 31 Rome. http://www.fao.org/docrep/006/ad665e/a d665e06.htm; https://fr.wikipedia.org/ wiki/Forêt,

Gonmadje CF, Donfack J-B, Kengué J. 2012. Rapport national sur l'état des ressources génétiques forestières $d u$ Cameroun. Organisation des Nations Unies pour l'Alimentation et l'Agriculture (FAO) ; 1-82.

Guillaumet J-L, Chevillotte H, Valton C. 2009. Les Forêts Tropicales Humides
Africaines, échelle 1:600 000, format $115 \times 75 \mathrm{~cm}$. IRD : Marseille, France.

Guinochet, M. 1973. Phytosociologie. Masson et Cie : Paris.

IUCN 2016. A global standard for the identification of Key Biodiversity Areas, Version 1.0, 1st ed. IUCN, Gland. https://portals.iucn.org/library/sites/librar y/files/documents/Rep-2016-005.pdf.

Letouzey R. 1968 Etude Phytogéographique du Cameroun. Ed. Paul Lechevalier. Paris Ve.

Letouzey R. 1985. Carte Phytogéographique du Cameroun au 1: 500 000, accompagnée de: Notices de la carte phytogéographique du Cameroun au 1: 500 000. Institut de la Carte Internationale de la Végétation. Toulouse. France.

Lézine A-M. 2008. Le Pollen. Outil d'Etude de l'Environnement et du Climat au Quaternaire. Société Géologique de France. Vuibert.

Lézine A-M, Holl A F-C, Lebamba J, Vincens A, Assi-Khaudjis C, Février L, Sultan E. 2013.Temporal relationship between Holocene human occupation and vegetation change along the northwestern margin of the Central African rainforest. C. R. Geoscience. http://dx.doi.org/10.1016/j.crte.2013.03. 001

Maley J. 1987. Fragmentation de la forêt dense humide africaine et extension des biotopes montagnards au Quaternaire récent: nouvelles données polliniques et chronologiques. Implications paléoclimatiques et biogéographiques. Paleontology of Africa, 18 : 307-334.

Maley J. 1990 L'histoire récente de la forêt dense humide africaine : essai sur le dynamisme de quelques formations forestières. In Paysages quaternaires de l'Afrique Centrale Atlantique, Lanfranchi R, Schwartz D (eds). Didactiques, ORSTOM. Paris; 383-388

Maley J. 1994. Le cadre paléoenvironnemental des refuges forestiers africains: quelques données et hypothèses. In The Bioderversity of African Plants, van der Maesen LJG, van der Burgt XM, van der 
Medenbach de Rooy (eds). Kluwer Academic Press; 519-535.

Maley J. 2001.La destruction catastrophique des forêts d'Afrique centrale survenue il y a environ 2500 ans exerce encore une influence majeure sur la répartition actuelle des formations végétales. Syst. Geogr. Pl., 71 : 777-796.

Mittermeier RA, Gil PR, Hoffman M, Pilgrim J, Brooks T, Mittermeier CG, Lamoreux J, Da Fonseca GAB. 2004. Hotspots Revised: Earth's Biologically Richest and most Threatened Ecoregions. CEMEX: Mexico City.

Moby EP. 1979. Climat. In Atlas de la République Unie du Cameroun, Laclavère $G$ (ed.). Les Editions J.A. : Paris; 16-19.

Momo S.M.C. 2009 Influence des activités anthropiques sur la végétation du mont Oku (Cameroun). Thèse de Doctorat en biologie-santé. Université de Picardie Jules Vernes et Université de Yaoundé I.

Momo SMC, Chabrerie O, Gallet-Moron E, Nkongmeneck B-A, Leumbe ONL, Decocq G. 2012. Analyse de la dynamique de déforestation par télédétection couplée aux modèles d'équations structurales: exemple de la forêt néphéliphile du mont Oku (Cameroun). Acta Botanica Gallica, 159 (4) : 451-466. DOI : http://dx.doi.org/10.1080/12538078.2012 .750583

Morin S. 1979a. Relief et hydrographie. In Atlas de la République Unie du Cameroun, Laclavère G (ed.). Les Editions J.A. : Paris; 5-8.

Morin S. 1979b. Géomorphologie. In Atlas de la République Unie du Cameroun, Laclavère $G$ (ed.). Les Editions J.A.: Paris; 13-15.

Morrone JJ. 2015. Biogeographical regionalisation of the world: a reappraisal. Australian Systematic Botany, 28: 81-90.

Neumann K, Bostoen K, Höhn A, Kahlheber S, Ngomanda A, Tchiengué B. 2012 First farmers in the Central African rainforest: A view from southern Cameroon. Quaternary International, 249: 53-62.
Neumann K, Eggert MKH, Oslisly R, Clist B, Denham $\mathrm{T}$, de Maret $\mathrm{P}$, Ozainne $\mathrm{S}$, Hildebrand E, Bostoen K, Salzmann U, Schwartz D, Eichhorn B, Tchiengué B, Höhn A. 2012. Comment on "Intensifying Weathering and Land Use in Iron Age Central Africa". Science, 337 (6098) : 1040. DOI: $10.1126 /$ science. 1221747

Ngako V. 2006. Le Cameroun. Géologie, ressources minières. In Atlas $d u$ Cameroun, Collection Atlas de l'Afrique. Ben Yamed D, Houstin N, Seignobos C. (eds). Les Editions J.A. : Paris ; 60-61.

Ngomanda A, Neumann K, Schweizer A, Maley J. 2009. Seasonality change and the third millennium BP rainforest crisis in southern Cameroon (Central Africa). Quaternary Research, 71: 307-318.

Ntoupka M. 1999. Impacts des perturbations anthropiques (pâturages, feu et coupe de bois) sur la dynamique de la savane arborée en zone soudano-sahélienne nord $\mathrm{du}$ Cameroun. Thèse de Doctorat. Université Paul Valery-Montpellier III.

Onana JM. 2010. Etat de connaissance de la flore du Cameroun. In Systématique et Conservation des Plantes Africaines, van der Burgt XM, van der Maesen LJG, Onana JM. (eds). Royal Botanic Gardens, Kew; 557-569.

Onana JM. 2013. Synopsis des espèces végétales vasculaires endémiques et rares du Cameroun. Check-liste pour la gestion durable et la conservation de la biodiversité. In Flore du Cameroun 40, Onana JM (ed.). MINRESI, Yaoundé.

Onana JM, Cheek M. 2011. Red Data Book of the Flowering Plants of Cameroon: IUCN global assessments. Royal Botanic Gardens, Kew and IRAD-National Herbarium of Cameroon. Kew Publishing: London.

Republic of Cameroon 2009. Fourth national report on biodiversity. Ministère de l'Environnement et des Forêts, Yaoundé.

Republic of Cameroon 2012. National Biodiversity Strategy and Plan Action Version II. Ministère de l'Environnement de la Protection de la nature et du Développement Durable, Yaoundé. 
République du Cameroun 2014. Cinquième Rapport National du Cameroun à la Convention de la Diversité Biologique. MINEPDED. URL: www.cbd.int

Secrétariat de la Convention sur la Diversité Biologique. 2004. Approche par Écosystème (Lignes Directrices de la $C D B)$. Montréal.

Tchatchou B, Sonwa DJ, Ifo S, Tiani AM. 2015. Déforestation et dégradation des forêts dans le Bassin du Congo : État des lieux, causes actuelles et perspectives. Papier occasionnel 120. Bogor, Indonésie. CIFOR.

http://www.cifor.org/publications/pdf_fil es/OccPapers/OP-120.pdf

Tchawa P. 2006. Le Cameroun. Relief et hydrographie. In Atlas du Cameroun, Collection Atlas de l'Afrique, Ben Yamed D, Houstin N, Seignobos C (eds). Les Editions J.A. : Paris; 58-59.

Tchouto MGP. 2004. Plant diversity in a central African rain forest: implications for biodiversity conservation in Cameroon. $\mathrm{PhD}$ Thesis. Department of plant Sciences, Biosystematics Group, Wageningen University, the Netherlands.

Tessa B. 2012. L'Atlas Forestier Interactif du Cameroun pour une meilleure gestion des ressources forestières. http://www.wri.org/blog/2012/10/1\%E2 $\% 80 \% 99$ atlas-forestier-interactif-ducameroun-pour-une-meilleure-gestiondes-ressources. 11 Octobre 2012.

Tsaléfac M. 2006. Le Cameroun. Climat. In Atlas du Cameroun, Collection Atlas de l'Afrique, Ben Yamed D, Houstin N, Seignobos C (eds). Les Editions J.A. : Paris; 62-63.

Unité Technique du Projet d'Inventaire forestier national, FAO 2006. Evaluation des ressources forestières nationales du Cameroun. Rapport. Ministère des Forêts et de la Faune, Organisation des Nations Unies pour l'Alimentation et l'Agriculture. République du Cameroun ; 1-233.
Van Germerden BS, Shu GN, Olff H. 2003. Recovery of conservation values in Central African rain forest after logging and shifting cultivation. Biodiv. Conser., 12: $1553-1570$.

Vivien J. 2012. Guide des Mammifères et Poissons du Cameroun. Nguila Kérou (J. Vivien). Imp. Ediprint : Paris.

Vivien J, Depierre D. 2012. Mammifères Sauvages du Cameroun. Nguila Kérou (J. Vivien). Imp. Ediprint, Paris.

White F. 1979. The Guineo-Congolian Region and its relationships to other phytochoria. Bull. Jard. Bot. Nat., 49: 11-55.

Williams PH, Gaston KJ, Humphries CJ. 1997. Mapping biodiversity value worldwide: Combining higher-taxon richness from different groups. Proceedings of the Royal Society of London Series B Biological Sciences, 264: 141-148.

Williams PH, Burgess ND, Rahbek C. 2000. Flagship species, ecological complementarity, and conserving the diversity of mammals and birds in subSaharan Africa. Animal Conservation, 3: 249-260.

Witté I., Touroult J. 2014 Répartition de la biodiversité en France métropolitaine : une synthèse des Atlas faunistiques, VertigO - la revue électronique en sciences de l'environnement, 14(1). http://vertigo.revues.org/14645; DOI: 10.4000/vertigo. 14645

World Ressources Institute, USAID-CARPE 2008. Domaine forestier du Cameroun, Décembre 2008. Carte. République du Cameroun.

WWF. 2017. List of terrestrial ecoregions (WWF). https://en.wikipedia.org/wiki/List_ of_terrestrial_ecoregions_(WWF). 